\title{
A well-being program for women leaders with stress complaints
}

\author{
Elisa H. Kozasa*1, Shirley S. Lacerda1, Carla R. C. Barrichello2, Cássia C. Coelhoso1, Patricia R. Tobo2 \\ *Presenting author: Av. Albert Einstein, 627, Sao Paulo, SP, Brazil, CEP 05692-900; phone: 11-99179-9721 \\ 1- Hospital Israelita Albert Einstein, São Paulo, SP, Brazil \\ 2- Natura Cosmetics- Cajamar, São Paulo, SP, Brazil
}

Objectives: The objective of this study was to evaluate a wellbeing protocol based on positive psychology principles and meditation for women leaders with stress complaints.

Background and aims: There are many studies about stress in the mental health of work-force employees, however a relatively small number of studies have been focused stressrelated symptoms in managers or leaders. Managers have been considered a high-risk group in developing stress because of their psychological demands and workloads. Women have been assuming higher positions in major companies, which exposes them to higher levels of stress (Fig. 1). Some studies have been indicating that women leaders are more likely to experience emotional stress or fatigue than men due to excessive empathy, therefore we aimed to evaluate a wellbeing program designed for this group.

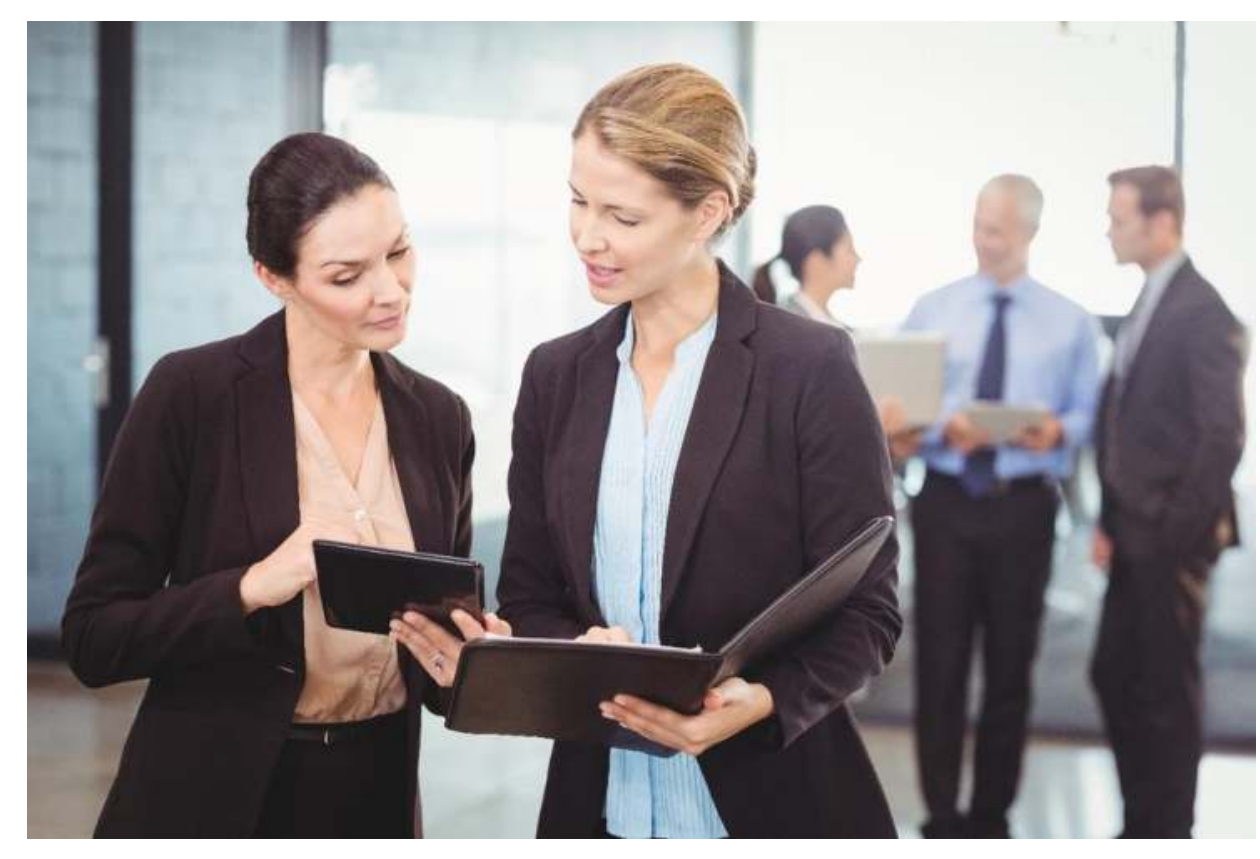

Fig. 1 Women have been assuming higher positions in companies.

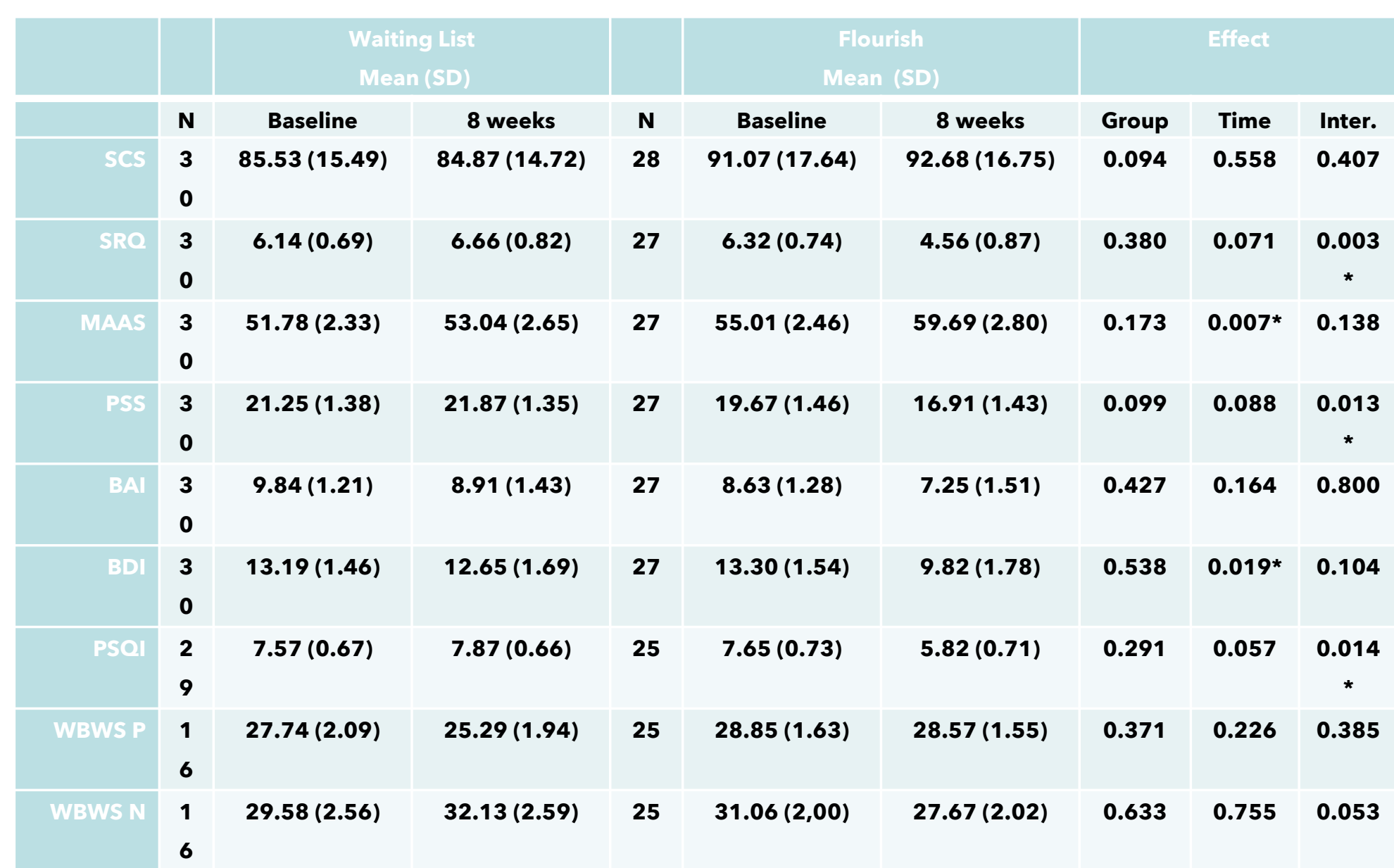

Materials and methods: Women sales and administrative managers in a multinational cosmetics company, with stress complaints, aged from 25 to 60 years, with at least university degree, were recruited to participate in a controlled trial evaluating a well-being program. They were allocated to the Control Group (CG- a waiting list group $\mathrm{N}=30$ ) or the Flourish Group (FG- intervention group $\mathrm{N}=27$ ). They were evaluated before and after 8 weeks in relation to their levels of stress, mindfulness, well-being at work, sleep, self-compassion, depression, anxiety, and self-reported mental health.

Results: There were no differences in baseline between groups in these variables. After 8 weeks the Flourish Group improved significantly compared to the Control Group in self-reported mental health (CG $6.66 \pm 0.82$ and FG $4.56 \pm 0.87$; $p=0.003$ ), stress (CG $21.87 \pm 1.35$ and FG $16.91 \pm 1.43 ; \quad p=0.013)$ and sleep symptoms (CG $7.87 \pm 0.66$ and FG 5.82 $\pm 0.71 ; p=0.014)$.

Conclusions: A combined positive psychology and meditation intervention (Flourish) may help women leaders to improve their mental health symptoms, sleep quality and perceived stress.

Funding: We would like to thank Natura Cosméticos S.A. and Instituto Israelita de Ensino e Pesquisa Albert Einstein for the financial support. 